\title{
First experimental evidence of the third conformer of glycolic acid: combined matrix isolation, FTIR and theoretical study
}

\author{
Igor D. Reva *, Susana Jarmelo, Leszek Lapinski ${ }^{1}$, Rui Fausto \\ Department of Chemistry, University of Coimbra, Rua Larga, 3004-535 Coimbra, Portugal
}

Received 21 January 2004; in final form 16 March 2004

\begin{abstract}
The FT-IR spectra of monomeric glycolic acid, GA, isolated in argon and xenon matrices (at 10 and $20 \mathrm{~K}$ ) were studied, enabling identification of three different conformers of GA. The most stable SSC conformer involves a hydrogen bond between the carbonyl group and the hydrogen atom of the alcohol hydroxyl group. The minor GAC and AAT forms involve intramolecular hydrogen bonds between alcohol and carboxyl $\mathrm{OH}$ groups, with alcohol and carboxylic groups serving as proton donors, respectively. The GAC conformer, predicted by theoretical calculations (at the DFT/B3LYP and MP2 levels with the 6-31++G(d,p) and aug-ccpVDZ basis sets) as the second most stable form, was now experimentally identified for the first time.

(C) 2004 Elsevier B.V. All rights reserved.
\end{abstract}

\section{Introduction}

Glycolic acid, GA, prototype of the series of $\alpha$-hydroxy carboxylic acids, is well known for its important role in dermatology and cosmetics industry, being used as inhibitor of harmful oxidation biochemical processes [1-4]. Important research is also presently going on in order to develop new materials based on biodegradable polymers derived from GA that can be used for reconstruction of biological tissues and in organ transplantation [5].

Being the simplest member of this family of molecules, GA is by far the most studied $\alpha$-hydroxy acid, both experimentally and theoretically. The structure of the crystal was found to consist of a loose threedimensional hydrogen bonded network of two closely similar but crystallographically distinct types of molecules $[6,7]$. The heavy atom frames in these molecules were found to be nearly planar with the alcoholic $\mathrm{OH}$ group involved in an internal hydrogen bond with the

\footnotetext{
${ }^{*}$ Corresponding author. Fax: +351-239827703.

E-mail address: reva@qui.uc.pt (I.D. Reva).

${ }^{1}$ Permanent address: Institute of Physics, Polish Academy of Sciences, Al. Lotnikow 32/46, 02668 Warsaw, Poland.
}

carbonyl group, and, in addition, participating in the intermolecular hydrogen bond network. The conformation assumed by individual GA molecules in the crystal was found to resemble the most stable conformer observed in the gas phase both by microwave spectroscopy $[8,9]$ and electron diffraction [10] (the SSC conformer shown in Fig. 1), though in the crystal intermolecular interactions lead to deviation of the alcoholic $\mathrm{OH}$ group a few degrees from the plane of the $\mathrm{CC}(=\mathrm{O}) \mathrm{OH}$ fragment. Theoretical calculations undertaken at different levels of theory [11-17] also predict the SSC conformer as the conformational ground state for monomeric GA.

In the electron diffraction study of Iijima and collaborators [10], the presence in the gaseous phase of a second conformer was postulated, though no direct assignment of that form could be done. Nevertheless, the authors estimated that a conformer differing from the most stable conformer by internal rotation around the $\mathrm{C}-\mathrm{C}$ bond (the GAC conformer shown in Fig. 1) should be present in the gas phase, at $396 \mathrm{~K}$, with a population less than $1 \%$, corresponding to an estimated energy difference to the conformational ground state of $\approx 18$ $\mathrm{kJ} \mathrm{mol}^{-1}$ [10]. The unequivocal identification of a species in the electron diffraction technique is inherently limited 
<smiles>CC(O)C(=O)O</smiles>

$\mathrm{SSC}$<smiles>CC(O)C(=O)O</smiles>

$\mathrm{SAC}$<smiles>CC(C)(O)C(=O)O</smiles>

GAC

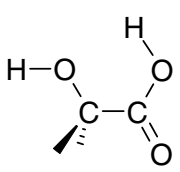

AAT
Fig. 1. Schematic representation of the conformer structures. Protons of the methylene group (at the alpha-carbon atom) are not shown explicitly. Conformers are denoted by the values of respective dihedral angles: $\mathrm{HO}_{\mathrm{A}} \mathrm{CC}$ (syn, anti, gauche); $\mathrm{O}_{\mathrm{A}} \mathrm{CC}=\mathrm{O}\left(\right.$ syn, anti); $\mathrm{O}=\mathrm{CO}_{\mathrm{C}} \mathrm{H}$ (cis, trans). To distinguish oxygen atoms of two $\mathrm{OH}$ groups, here subscripts ${ }_{\mathrm{A}}$ and $\mathrm{C}_{\mathrm{C}}$ denote that the oxygen atom belongs to the alcohol (A) or carboxylic (C) fragment. Thus all possible structures are denoted by three-letter code, e.g. SSC, GAC, AAT (syn-syn-cis, gaucheanti-cis, anti-anti-trans, respectively).

to species differing from the others by positions of heavy atoms. The species differing only by positions of labile protons have similar signatures in the electron diffraction experiments. The analysis of Fig. 1 shows that, in the case of GA, the SAC, GAC and AAT structures have similar heavy atom arrangements. This explains why a definite conclusion about the structure of the minor form could not be reached [10]. An analogous situation has been previously reported for glycine. The most stable glycine conformer was easily identified by electron diffraction [18], which however could not distinguish between the second and the third forms which have an identical heavy atom backbone.

A more recent microwave spectroscopic study [17], in addition to the most stable SSC form, could also identify the presence of another conformer in the jet cooled GA (the AAT conformer shown in Fig. 1). The failure to observe experimentally the second most stable conformer of monomeric GA (the GAC form) in that study was explained [17] in terms of the pathways and energy barriers involved in the possible conformational interconversions predicted theoretically $[13,17]$. It was suggested that the second conformer would relax in the jet expansion to the most stable conformer, since the energy barrier separating these two forms is low enough $(\approx 11$ $\mathrm{kJ} \mathrm{mol}^{-1}$ ) [17] to be overcome, thus making impossible the experimental observation of the GAC form. On the other hand, the AAT conformer cannot convert either to SSC or GAC forms during expansion [17], due to the high energy barriers associated with these processes, thus being observed experimentally.

There is, however, an alternative explanation for the non-observation of the GAC form in the microwave experiments. The intensities of transitions in microwave experiments are proportional to the square of the molecular dipole moment. Of the three most stable GA conformers, the second most stable conformer, GAC, possesses the smallest dipole moment (calculated $\operatorname{CCSD}(\mathrm{T}) / 6-31 \mathrm{G}(\mathrm{d}, \mathrm{p}) / / \mathrm{B} 3 \mathrm{LYP} / 6-311+\mathrm{G}(\mathrm{d}, \mathrm{p})$ values are 2.44, 2.03 and $5.45 \mathrm{D}$ for the SSC, GAC and AAT, respectively) [16] and then represents the most difficult species to be detected in the microwave spectra. An analogous situation has been previously reported for glycine. The microwave experiments where able to identify two of the three stablest glycine conformers. Chronologically, the third (in terms of relative energy) glycine conformer, with the highest dipole moment (5.45 D) [19] was registered at the earliest [20]. Later, the first (most stable) conformer (dipole moment 1.15 D) [19] could be identified [21] with a considerable experimental effort. The second glycine conformer (predicted dipole moment $\approx 2$ D) [22] could not be identified at all. The analogy between glycolic acid and glycine seems to be obvious.

It only remains to say that the 'missing' second glycine conformer, not observable either in microwave or electron diffraction experiments, could be successfully identified by matrix isolation infrared spectroscopy, along with the other two forms [22]. Thus, the use of matrix-isolation FTIR spectroscopy for conformational analysis of glycolic acid, by analogy with the glycine case, appeared to be very promising.

Hollenstein and collaborators $[23,24]$ investigated the infrared spectra of several isotopologues of GA isolated in argon. Besides the dominant spectral features present in the freshly prepared matrix, ascribable to the most stable SSC conformer, they were able to observe, in the spectrum obtained after broadband infrared irradiation of the matrix, a series of new bands which were assigned to the third most stable conformer predicted theoretically $[13,14,23,24]$. Surprisingly, the presence in the matrix samples of a third conformational structure was not deduced. The lack of observation of the GAC conformer cannot be easily explained in the case of the matrix isolation studies [23,24]. In fact, infrared induced direct conversion from the most stable conformer to AAT did not appear to be possible (the energy barrier is higher than $75 \mathrm{~kJ} \mathrm{~mol}^{-1}$ ) $[13,17,24]$. The most probable pathway for this rotamerization involves conversion from SSC to GAC and then from this form to AAT [24], the predicted energy barriers for these two processes being respectively $\approx 20$ and $40 \mathrm{~kJ} \mathrm{~mol}^{-1}$ [24]. This means that, if this is the preferred reaction channel to the proposed photoisomerization, form GAC should also be detected, at least during the initial stages of the reaction, unless the mechanism for intramolecular energy redistribution is much more efficient for the last step than for the first one. Another possibility is that the newly appearing bands in the spectrum of the irradiated matrix [24] were due to both GAC and AAT conformers. To investigate this possibility, however, it would be essential to identify the vibrational signatures of matrixisolated GAC and AAT conformers.

Photoexcitation induces changes in relative conformational populations after matrix deposition. Alternatively, the populations of the GAC and AAT conformers, to be trapped in a low temperature matrix, 
may be increased thermally in the gaseous phase, prior to deposition. In the present study we explored this idea, taking advantage of the deposition cell we have developed in our laboratory [25], which has already enabled a successful identification of thermally populated minor conformers of pyruvic [25] and lactic [26] acids.

\section{Experimental and computational methods}

Glycolic acid of spectroscopic grade purity was obtained from Aldrich and additionally purified from volatile impurities by pumping via cryostat immediately before experiments. A glass vacuum system and standard manometric procedures were used to deposit matrix gases (Air Liquide, argon, 99.9999\%; xenon, $99.995 \%$ ), which were used without further purification. Gas deposition rate during sample preparation was $\approx 10 \mathrm{mmol} \mathrm{h}^{-1}$. GA was co-deposited from a specially designed Knudsen cell with shut-off possibility, the principal component of which is a SS-4BMRG micrometer valve (NUPRO). The cell has two independent thermostattable parts, the valve nozzle and the sample compartment. This design enables an independent control of the saturated gas pressure over the compound (by the choice of temperature of the sample compartment) and control of the equilibrium conformational populations (by variation of the nozzle temperature). Further details of the experimental setup can be found in [25].

The infrared spectra in the range $4000-400 \mathrm{~cm}^{-1}$ were obtained using a Mattson (Infinity 60AR Series) Fourier transform infrared spectrometer equipped with a deuterated triglycine sulphate (DTGS) detector and $\mathrm{Ge} / \mathrm{KBr}$ beamsplitter. Data collection was performed with $0.5 \mathrm{~cm}^{-1}$ spectral resolution. An effort was made to avoid association of glycolic acid in matrices. The characteristic absorptions of associates (e.g. in the carbonyl stretching region those are red-shifted by $\approx 50$ $\mathrm{cm}^{-1}$ with respect to the monomer bands) were monitored during deposition and annealing of the samples. It was assured that the freshly deposited matrices contained essentially monomeric GA. All experiments were done based on an APD Cryogenics closed-cycle helium refrigeration system with a DE-202A expander.

The theoretical calculations were performed with the GAUSSIAN 98 program package [27] at both DFT and MP2 levels with the standard 6-31++G(d,p) and aug-ccpVDZ basis sets $[28,29]$. The DFT calculations were carried out with the three-parameter density functional abbreviated as B3LYP, which includes Becke's gradient exchange correction [30], the Lee, Yang, Parr correlation functional [31] and the Vosko, Wilk and Nusair correlation functional [32]. The geometry optimizations were followed by frequency calculations to check the nature of the calculated structures. The calculated frequencies were used to assist the analysis of the experi- mental spectra and to account for the zero-point vibrational energy (ZPVE) corrections (without scaling). The best correspondence between the experimentally observed and the theoretically predicted spectra was obtained for the MP2/aug-cc-pVDZ level of theory. The MP2/aug-cc-pVDZ calculated frequencies were scaled using two scaling factors: 0.993 below $2000 \mathrm{~cm}^{-1}$ and 0.955 above $2000 \mathrm{~cm}^{-1}$ (i.e. for $\mathrm{OH}$ str). These scaling factors give the best agreement between the predicted and observed frequencies (in argon matrix), especially in the $\mathrm{OH}$ stretching and $\mathrm{C}=\mathrm{O}$ stretching regions, and the MP2/aug-cc-pVDZ scaled frequencies will be cited throughout the work.

\section{Results and discussion}

There are three internal degrees of freedom in glycolic acid and possible conformers can be defined by the values of the three dihedral angles: $\mathrm{HO}_{\mathrm{A}} \mathrm{CC}$ (syn, anti, gauche); $\mathrm{O}_{\mathrm{A}} \mathrm{CC}=\mathrm{O}$ (syn, anti); $\mathrm{O}=\mathrm{CO}_{\mathrm{C}} \mathrm{H}$ (cis, trans), where (A) denotes alcoholic $\mathrm{OH}$ group and (C) the $\mathrm{OH}$ group in the carboxylic fragment. There are eight possible GA structures with a planar arrangement of the heavy atom framework. One of these structures (SAT) has a crowded geometry, with the hydrogen atoms of the two $\mathrm{OH}$ groups directed towards each other. The calculations show that this is a second order saddle point and has a very high relative energy $\left(50-60 \mathrm{~kJ} \mathrm{~mol}^{-1}\right)$. Thus, this form is excluded from the following analysis. The remaining seven structures have been optimized with explicit symmetry $\left(\mathrm{C}_{\mathrm{s}}\right)$. Depending on the level of the calculations, the optimized structures exhibit either zero (minima) or one (first order saddle point) negative frequency at the explicit $\mathrm{C}_{\mathrm{s}}$ geometry. However, as follows from the optimizations without symmetry restrictions, all but one planar first order saddle points have related neighboring non-planar minima differing in energy from these structures not more than tenths of $\mathrm{kJ} \mathrm{mol}^{-1}$. Only the GAC conformer exhibits clear nonplanarity at any of the applied calculation levels. The calculated energy difference between the planar, first order saddle point structure SAC and the two mirror GAC conformations is $4.4 \mathrm{~kJ} \mathrm{~mol}^{-1}$ (at the MP2/ $6-31++\mathrm{G}(\mathrm{d}, \mathrm{p})$ level) or $2.6 \mathrm{~kJ} \mathrm{~mol}^{-1}$ (at the MP2/aug-ccpVDZ level). The corresponding potential energy profile is presented in Fig. 2. The imaginary mode at the planar saddle point (SAC) corresponds to $\mathrm{HO}_{\mathrm{A}} \mathrm{CC}$ torsion and its frequency (calculated at the MP2/6-31++G(d,p) level) is equal to 282 (i) $\mathrm{cm}^{-1}$ (195 (i) $\mathrm{cm}^{-1}$ at the MP2/ aug-cc-pVDZ level). The frequency of the corresponding vibrational mode in the potential well minimum (GAC form) is equal to 356 and $322 \mathrm{~cm}^{-1}$ at the MP2/ 6-31++G(d,p) and MP2/aug-cc-pVDZ levels, respectively. This implies a zero-point energy of 2.1 and 1.9 $\mathrm{kJ} \mathrm{mol}^{-1}$, still below the barrier. 


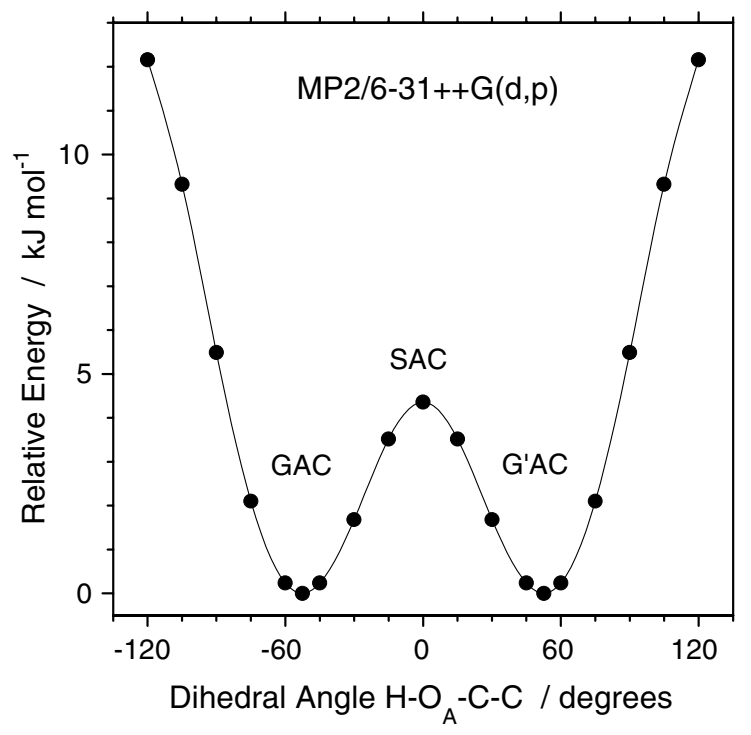

Fig. 2. Barrier for internal rotation connecting the non-planar GAC form with its mirror image (G'AC). Top-of-the-barrier planar $\left(\mathrm{C}_{\mathrm{s}}\right)$ structure is a first-order saddle point and corresponds to the SAC geometry. Calculation at the MP2/6-31++G(d,p) level of theory. Energy of the GAC form is assumed as zero-level. Zero-point vibrational energy is not included.

In consonance with the literature data [12-17], the SSC conformer was predicted at all levels of calculation as the global energy minimum. The relative energies of the other forms at different calculation levels are summarized in Table 1.

At the highest of the used levels of theory (MP2/augcc-pVDZ), five geometries (including SSC) with a planar heavy atom framework were found to be true minima on the potential energy surface, while the GAC and AAT forms kept non-planarity of the heavy atoms in the geometries corresponding to the minima. For the AAT form, the calculated difference in energies between the non-planar and the planar structures is $0.003 \mathrm{~kJ} \mathrm{~mol}^{-1}$ only (at the MP2/aug-cc-pVDZ level).

Taken into consideration the topology of the potential energy surface around the different minima and the calculated relative energies estimated at the MP2/aug-ccpVDZ level of theory (Table 1), the populations of glycolic acid conformers were estimated for the equilibrium gaseous phase at different temperatures, using the Boltzmann distribution. The GAC conformer was considered as doubly degenerated by symmetry (see Fig. 2), while all the other conformers were assumed to have the statistical weights of unity. At room temperature $(297 \mathrm{~K})$, the equilibrium mixture contains mainly two forms, SSC (95\%) and GAC (4\%), while, altogether, the remaining conformers contribute less than $1 \%$ to the total population. Further, within the temperature range at which the compound does not decompose (below $200{ }^{\circ} \mathrm{C}$ ) only three forms (SSC, GAC and AAT) contribute noticeably to the equilibrium. The populations of these forms at $428 \mathrm{~K}$ are theoretically predicted to be equal $83.8 \%, 11.7 \%$ and $2.9 \%$, for SSC, GAC and AAT, respectively. All other forms represent less than $2 \%$ of the population (Table 1).

As mentioned in the introduction, the lowest energy conformer (SSC) and the AAT form have been observed experimentally before. Very interestingly, in the equilibrium at room temperature 1\% of GA should appear in the AAT form and at $428 \mathrm{~K}$ this value should be only $3 \%$, i.e. much less than the contribution of the GAC form, which should vary from $4 \%$ to $12 \%$ in the same temperature range. Thus, according to the theoretical expectations, the GAC form should be present in the gas phase, already at moderately elevated temperatures, in an amount sufficient for the experimental registration. Remarkably, the GAC form has not been observed

Table 1

Energies relative to the SSC form (ZPVE included); energies of the SSC form (ZPVE included); dipole moments calculated at different levels of theory and equilibrium Boltzmann populations of different glycolic acid conformers, calculated for the relative energies predicted at the MP2/aug-cc-pVDZ level of theory and temperatures used in the present study

\begin{tabular}{|c|c|c|c|c|c|c|c|c|}
\hline & $\mathrm{SSC}$ & GAC & AAT & ASC & $\mathrm{AAC}$ & SST & AST & SSC \\
\hline Calculation method & \multicolumn{7}{|c|}{ Relative energies $\left(\mathrm{kJ} \mathrm{mol}^{-1}\right)$} & Energies (Hartrees) \\
\hline B3LYP/6-31++G(d,p) & 0.00 & 9.38 & 12.86 & 18.77 & 19.60 & 22.66 & 45.83 & -304.253801 \\
\hline MP2/6-31++G(d,p) & 0.00 & 7.92 & 13.28 & 17.25 & 19.59 & 24.73 & 47.11 & -303.435695 \\
\hline B3LYP/aug-cc-pVDZ & 0.00 & 10.54 & 13.04 & 17.80 & 19.05 & 19.20 & 41.15 & -304.285158 \\
\hline \multirow[t]{2}{*}{ MP2/aug-cc-pVDZ } & 0.00 & 9.48 & 12.03 & 16.60 & 18.60 & 19.11 & 40.12 & -303.517652 \\
\hline & \multicolumn{7}{|c|}{ Dipole moments (Debye) } & \\
\hline B3LYP/6-31++G(d,p) & 2.45 & 1.97 & 5.16 & 3.29 & 1.82 & 3.62 & 6.02 & \\
\hline $\mathrm{MP} 2 / 6-31++\mathrm{G}(\mathrm{d}, \mathrm{p})$ & 2.57 & 2.19 & 5.64 & 3.71 & 2.40 & 4.01 & 6.54 & \\
\hline B3LYP/aug-cc-pVDZ & 2.31 & 1.85 & 4.95 & 3.16 & 1.79 & 3.48 & 5.67 & \\
\hline MP2/aug-cc-pVDZ & 2.44 & 2.02 & 5.47 & 3.56 & 2.08 & 3.86 & 6.31 & \\
\hline Temperature & \multicolumn{8}{|c|}{ Equilibrium Boltzmann populations (\%) } \\
\hline $297 \mathrm{~K}$ & 94.98 & 4.09 & 0.73 & 0.11 & 0.05 & 0.04 & 0.00 & \\
\hline $428 \mathrm{~K}$ & 83.83 & 11.69 & 2.85 & 0.79 & 0.45 & 0.39 & 0.00 & \\
\hline
\end{tabular}


previously, while AAT was, following photochemical production from the conformational ground state [24], as mentioned in Section 1.

In order to search for the missing conformer, we opted for isolating GA in a low temperature argon matrix using the gaseous compound heated to moderate temperatures (to avoid thermal decomposition) and keeping the temperature of the cold substrate of the cryostat as low as possible.

The $1820-1750 \mathrm{~cm}^{-1}$ region of the infrared spectra of GA isolated in Ar and Xe matrices is presented in Fig. 3. The bands due to the stretching vibrations of the carbonyl group appear in this region. The observation that the relative intensities of the bands in this region are different in matrices obtained by deposition of GA from the nozzle at 297 and $428 \mathrm{~K}$ clearly shows that more than one conformer of the compound was trapped in the solid noble gas. Upon increasing the temperature of gaseous GA, the populations of higher energy forms (GAC and AAT) should increase with respect to the population of the most stable form SSC. The experimental observations presented in Fig. 3 are in full

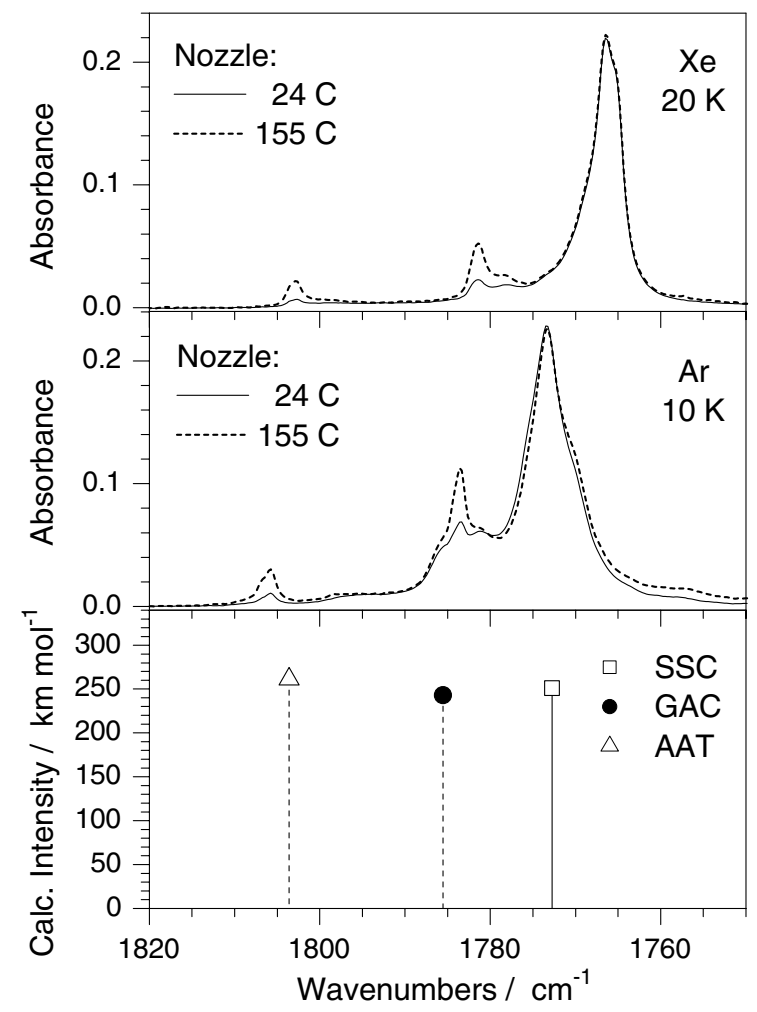

Fig. 3. Carbonyl stretching region. Lower frame represents results of calculations performed at the MP2/aug-cc-pVDZ level of theory. Calculated frequencies are scaled by 0.993. Experimental spectra (middle and upper frame): Solid line - nozzle at room temperature $(297 \mathrm{~K})$, dashed line - nozzle at $428 \mathrm{~K}$ during matrix deposition. Experimental spectra are normalized to the same intensity due to the peaks of the main (SSC) form. Upper frame: glycolic acid isolated in a xenon matrix at $20 \mathrm{~K}$; middle frame: glycolic acid isolated in an argon matrix at $10 \mathrm{~K}$. agreement with these expectations. This allows for the conclusion that the bands at $1805.8 \mathrm{~cm}^{-1}$ (Ar) and $1783.6 \mathrm{~cm}^{-1}$ (Ar) are spectral indications of the presence in the matrix of conformers other than the dominating SSC. Comparison of the experimental spectra with the results of theoretical calculations strongly suggests that these two bands should be assigned to the conformers AAT and GAC, respectively. Observation of the three forms in the xenon matrix, at $20 \mathrm{~K}$, with approximately the same relative amounts as in argon at $10 \mathrm{~K}$, indicates that the barriers to the interconformational rearrangements in the matrices are relatively high. In addition, it also implies that no conformational cooling occurs upon matrix preparation [33], the populations of the conformers in the freshly prepared matrices reflecting those present in the gaseous phase.

The vibrational signature of the three conformers, including the 'missing' GAC form, is also clearly identifiable in other spectral regions. In consonance with the previous matrix studies $[23,24]$, the present experiment shows that the two $\mathrm{OH}$ stretching vibrations of the dominant conformer (SSC) are situated at approximately the same frequency. This result is also confirmed by the calculations. The two minor forms became well discernible at a moderate heating of the gaseous phase (Fig. 4). The two expected $\mathrm{OH}$ stretching bands due to GAC (predicted at 3644.3 and $3569.3 \mathrm{~cm}^{-1}$, for $\mathrm{O}_{\mathrm{A}} \mathrm{H}$ and $\mathrm{O}_{\mathrm{C}} \mathrm{H}$, respectively), are observed as a doublet at $3653.5 / 3648.0 \mathrm{~cm}^{-1}$ and a band at $3573.5 \mathrm{~cm}^{-1}$. The latter band is clearly observed under the conditions of the present experiment, despite it almost coincides with the strong band of the main form, observed at 3566$3556 \mathrm{~cm}^{-1}$ (and predicted at 3563.2 and 3570.6 for $\mathrm{O}_{\mathrm{A}} \mathrm{H}$ and $\mathrm{O}_{\mathrm{C}} \mathrm{H}$, respectively). The coincidence of one of the $\mathrm{OH}$ bands due to the GAC form with the two intense bands due to SSC could have been one of the reasons why this conformer escaped from experimental identification in the previous matrix isolation studies $[23,24]$.

The bands due to AAT are observed at 3670.0 and $3473.2 \mathrm{~cm}^{-1}$, the latter being considerably overestimated by the calculations (calculated value: $3511.2 \mathrm{~cm}^{-1}$ ), as it generally occurs for vibrations of internally $\mathrm{H}$-bonded $\mathrm{OH}$ groups [34,35].

The bands corresponding to $\mathrm{C}=\mathrm{O}$ and $\mathrm{OH}$ stretching vibrations are obvious choices for searching for spectral manifestations of the minor conformers, due to the fact that these bands appear in relatively clean regions. Another interesting spectral range is that between 1100 and $800 \mathrm{~cm}^{-1}$ (Fig. 5). The IR bands of the minor conformer GAC present in this region can be observed and identified, because they do not overlap with the bands due to the AAT and SSC forms.

Note that the inset in the upper frame of Fig. 5 clearly shows that the absorptions due to the two minor forms are very weak (in the absolute scale). The dashed line in the inset corresponds to the maximum ordinate of the 


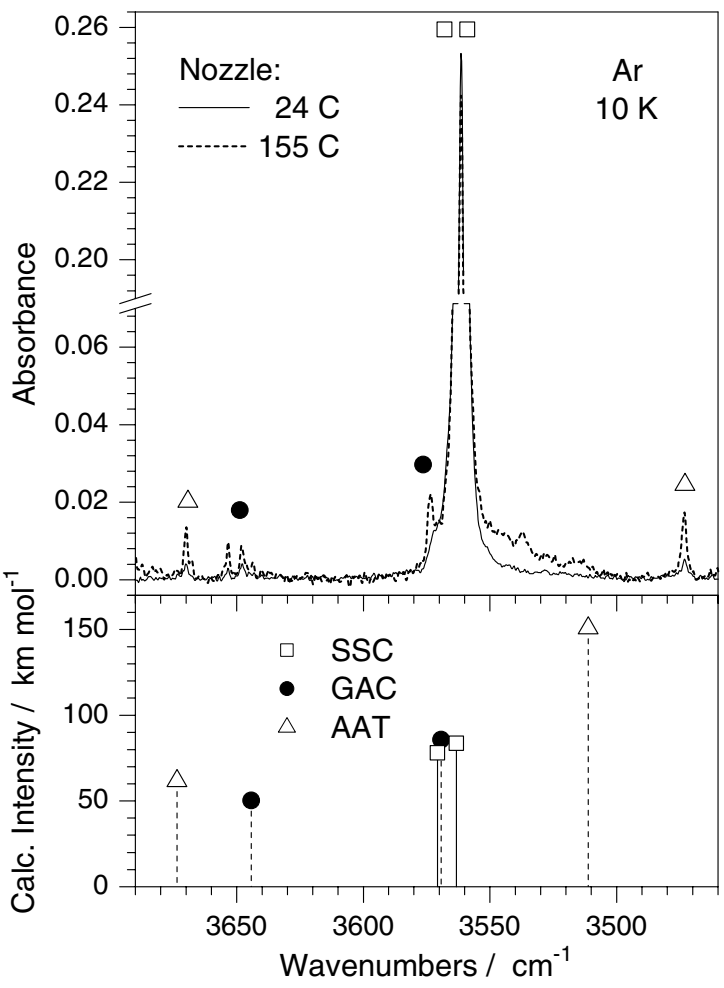

Fig. 4. OH stretching region. Lower frame represents results of calculations performed at the MP2/aug-cc-pVDZ level of theory. Calculated frequencies are scaled by 0.955 . Upper frame: glycolic acid isolated in an argon matrix at $10 \mathrm{~K}$. Solid line - nozzle at room temperature $\left(24{ }^{\circ} \mathrm{C}\right)$, dashed line - nozzle at $155^{\circ} \mathrm{C}$ during matrix deposition. Experimental spectra are normalized to the same intensity due to the peaks of the main (SSC) form. Note ordinate break in the experimental spectrum.

main frame. Hence, because of the weakness of the bands due to minor forms, special effort was done to accumulate the spectra and assure that the absorptions due to these forms are well above the noise level. In this spectral region, the assignment of the bands due to the main form is straightforward. In addition, the assignment of the vibrations due to AAT was also made easily, taking into account the results of the previously reported matrix isolation study of GA [24], where the AAT conformer was populated upon infrared irradiation of the matrix. Indeed, the assignments here made for the vibrations of AAT match both experimental data published before and the high-level calculations performed in the present study. This gives us some confidence in the identification of the bands due to the new minor form (GAC) based on the comparison of our experimental data with the theoretical predictions. The absorption bands at 1080, 997 and $825 \mathrm{~cm}^{-1}$, growing in the spectrum of the sample with the heated nozzle, match well the predicted frequencies 1080.9, 997.6 and $837.6 \mathrm{~cm}^{-1}$. The corresponding vibrations may be approximately described as $\left(\mathrm{C}-\mathrm{O}_{\mathrm{A}}\right.$ stretching $/ \mathrm{CO}_{\mathrm{A}} \mathrm{H}$

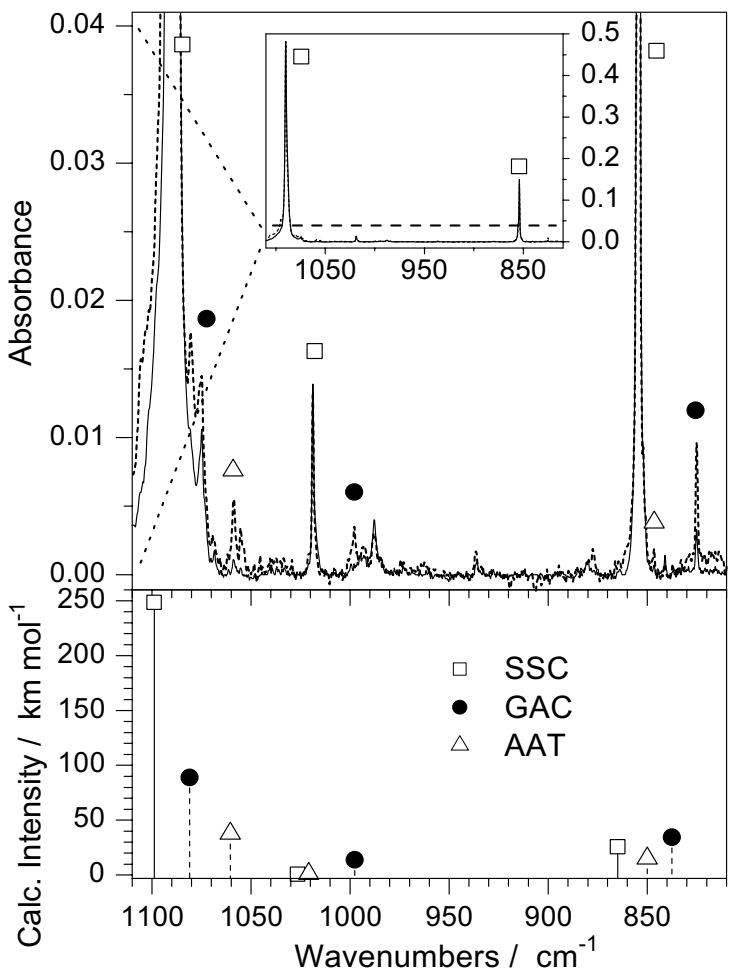

Fig. 5. Fingerprint region. Lower frame represents results of calculations performed at the MP2/aug-cc-pVDZ level of theory. Calculated frequencies are scaled by 0.993 . Upper frame: glycolic acid isolated in an argon matrix at $10 \mathrm{~K}$. Solid line - nozzle at room temperature $\left(24{ }^{\circ} \mathrm{C}\right)$, dashed line - nozzle at $155{ }^{\circ} \mathrm{C}$ during matrix deposition. Experimental spectra are normalized to the same intensity due to the peaks of the main (SSC) form. Horizontal dashed line in the inset frame represents the ordinate scale in the main upper frame.

bending $), \mathrm{CH}_{2}$ rocking and $\left(\mathrm{CC}\right.$ stretching $/ \mathrm{CO}_{\mathrm{C}}$ stretching) modes of GAC, respectively.

Assuming that the ratios of the conformers trapped in the low-temperature matrices correspond to the gas phase equilibrium at the temperature of the nozzle, the relative enthalpies of GA conformers can be derived (for the exact formula see our previous work [25]) on the basis of the change of integral band intensities as a function of the nozzle temperature. For this purpose, the strong bands due to the stretching vibrations of the carbonyl groups are especially useful. In the case of the argon matrix, the absorption of the GAC form develops on the shoulder of the absorption band due to the dominant SSC form, preventing a straightforward choice of the baseline. Thus, estimation of the relative enthalpy is only possible for AAT, yielding a value of about $10 \mathrm{~kJ} \mathrm{~mol}^{-1}$. For GA isolated in xenon matrices, the overlap between the bands is not much pronounced. In this case the intensities of these bands can be quite accurately measured. Based on the data from xenon matrices (deposition at 297 and $428 \mathrm{~K}$ ), the relative enthalpies of the AAT and GAC conformers were estimated as 12 and $9.6 \mathrm{~kJ} \mathrm{~mol}^{-1}$, with respect to the 
enthalpy of the SSC form. It should be emphasized that the relative enthalpies derived from the experimental data were obtained without recourse to any statistical factors. In the applied method the statistical factors are cancelled by division. The accuracy of this estimation is about $15-20 \%$, the main uncertainty being conditioned by the choice of the baseline for the bands ascribed to the minor conformers.

\section{Conclusion}

Conformers of glycolic acid were investigated using experimental low temperature matrix isolation technique as well as theoretical methods of quantum chemistry. Three conformers of the compound were observed and identified in argon or xenon matrices. In the most stable SSC conformer, the HOCC, $\mathrm{OCC}=\mathrm{O}$ and $\mathrm{O}=\mathrm{COH}$ dihedral angles are equal to $0^{\circ}$. In the less stable AAT and GAC forms, these three conformationally relevant dihedral angles are $170.88^{\circ}, 176.74^{\circ}$ and $179.90^{\circ}$, and $49.25^{\circ}, 156.22^{\circ}$ and $0.34^{\circ}$, respectively (calculated at the MP2/aug-cc-pVDZ level). The GAC form is experimentally identified for the first time. The relative energies of the three conformers were estimated on the basis of the relation between the temperature of the gaseous glycolic acid and the populations of its conformers trapped from the gas phase into a lowtemperature matrix.

\section{Acknowledgements}

The authors acknowledge Fundação para a Ciência e a Tecnologia (FCT), Lisbon (research project POCTI/ QUI/43366/2001 and Grants SFRH/BD/6696/2001 and SFRH/BPD/1661/2000) and FEDER, for financial support.

\section{References}

[1] L.S. Moy, K. Howe, R.L. Moy, Dermatol. Surg. 22 (1996) 439.

[2] L.S. Moy, H. Murad, R.L. Moy, J. Dermatol. Surg. Oncol. 19 (1993) 243.

[3] R.G. Males, F.G. Herring, Biochim. Biophys. Acta-Biomembr. 1416 (1999) 333.
[4] J.C. DiNardo, G.L. Grove, L.S. Moy, Dermatol. Surg. 22 (1996) 421.

[5] D.J. Mooney, C.L. Mazzoni, C. Breuer, K. McNamara, D. Hern, J.P. Vacanti, R. Langer, Biomaterials 17 (1996) 115.

[6] R.D. Ellison, C.K. Johnson, H.A. Levy, Acta Crystallogr. Section B - Struct. Crystallogr. Cryst. Chem. B 27 (1971) 333.

[7] W.P. Pijper, Acta Crystallogr. Section B - Struct. Crystallogr. Cryst. Chem. B 27 (1971) 344.

[8] H. Hasegawa, O. Ohashi, I. Yamaguchi, J. Mol. Struct. 82 (1982) 205.

[9] C.E. Blom, A. Bauder, J. Am. Chem. Soc. 104 (1982) 2993.

[10] K. Iijima, M. Kato, B. Beagley, J. Mol. Struct. 295 (1993) 289.

[11] M.D. Newton, G.A. Jeffrey, J. Am. Chem. Soc. 99 (1977) 2413.

[12] T.-K. Ha, C.E. Blom, H.H. Günthard, Theochem. J. Mol. Struct. 2 (1981) 285.

[13] M. Flock, M. Ramek, Int. J. Quantum Chem., Quantum Chemistry Symposium, 1992, p. 505.

[14] F. Jensen, Acta Chem. Scand. 51 (1997) 439.

[15] C. Chen, F.S. Hsu, Theochem. J. Mol. Struct. 506 (2000) 147.

[16] A.C. Tsipis, C.A. Tsipis, V. Valla, Theochem. J. Mol. Struct. 630 (2003) 81.

[17] P.D. Godfrey, F.M. Rodgers, R.D. Brown, J. Am. Chem. Soc. 119 (1997) 2232.

[18] K. Iijima, K. Tanaka, S. Onuma, J. Mol. Struct. 246 (1991) 257.

[19] F.J. Lovas, Y. Kawashima, J.U. Grabow, R.D. Suenram, G.T. Fraser, E. Hirota, Astrophys. J. 455 (1995) L201.

[20] R.D. Suenram, F.J. Lovas, J. Molec. Spectrosc. 72 (1978) 372.

[21] R.D. Suenram, F.J. Lovas, J. Am. Chem. Soc. 102 (1980) 7180.

[22] S.G. Stepanian, I.D. Reva, E.D. Radchenko, M.T.S. Rosado, M. Duarte, R. Fausto, L. Adamowicz, J. Phys. Chem. A 102 (1998) 1041.

[23] H. Hollenstein, R.W. Schär, N. Schwizgebel, G. Grassi, H.H. Günthard, Spectrochim. Acta Part A - Molec. Spectrosc. 39 (1983) 193.

[24] H. Hollenstein, T.-K. Ha, H.H. Günthard, J. Mol. Struct. 146 (1986) 289.

[25] I.D. Reva, S.G. Stepanian, L. Adamowicz, R. Fausto, J. Phys. Chem. A 105 (2001) 4773.

[26] A. Borba, A. Gómez-Zavaglia, L. Lapinski, R. Fausto, Phys. Chem. Chem. Phys. 6 (2004), Doi: 10.1039/B316642B.

[27] M.J. Frisch et al., Gaussian 98, Revision A.9, Pittsburgh, PA, 1998.

[28] M.J. Frisch, J.A. Pople, J.S. Binkley, J. Chem. Phys. 80 (1984) 3265.

[29] R.A. Kendall, T.H. Dunning Jr, R.J. Harrison, J. Chem. Phys. 96 (1992) 6796.

[30] A.D. Becke, Phys. Rev. A 38 (1988) 3098.

[31] C.T. Lee, W.T. Yang, R.G. Parr, Phys. Rev. B 37 (1988) 785.

[32] S.H. Vosko, L. Wilk, M. Nusair, Can. J. Phys. 58 (1980) 1200.

[33] I.D. Reva, S.G. Stepanian, L. Adamowicz, R. Fausto, Chem. Phys. Lett. 374 (2003) 631.

[34] E.M.S. Maçôas, R. Fausto, J. Lundell, M. Pettersson, L. Khriachtchev, M. Räsänen, J. Phys. Chem. A 104 (2000) 11725.

[35] E.M.S. Maçôas, R. Fausto, J. Lundell, M. Pettersson, L. Khriachtchev, M. Räsänen, J. Phys. Chem. A 105 (2001) 3922. 